\title{
Andersen-Tawil syndrome (ATS) - Case report and literature review
}

\author{
Katarzyna Olszewska', Jerzy Błaszczak', Maria Mielnik-Błaszczak ${ }^{2}$ \\ ${ }^{1}$ Chair and Department of Jaw Orthopaedics, Medical University in Lublin, Poland \\ ${ }^{2}$ Chair and Department of Paediatric Dentistry, Medical University in Lublin, Poland
}

Olszewska K, Błaszczak J, Mielnik-Błaszczak M. Andersen-Tawil syndrome (ATS) - Case report and literature review. J Pre-Clin Clin Res. 2014; 8(2): 123-126. doi: 10.5604/18982395.1135665

\begin{abstract}
Andersen-Tawil Syndrome (ATS) is a rare genetic disorder inherited in an autosomal dominant pattern caused by mutations in the KCNJ2 gene encoding Kir2.1 protein forming potassium ion channel, leading to disruption of cardiac and skeletal muscle repolarisation. Clinical symptoms include periodic paralysis, ventricular arrhythmia associated with QT prolongation and typical skeletal and facial dysmorphic features. The aim of the study was to present characteristic features of the rare Andersen-Tawil syndrome (ATS) within the face and oral cavity of a 9-year-old boy. The patient was diagnosed with Andersen-Tawil syndrome (OMIM\#170390) at the age of 8 due to the positive family history, typical dysmorphic features, and the presence of mutation in the KCNJ2 gene confirmed by genetic testing. Typical manifestations of ATS were diagnosed: cardiac arrhythmia, short stature, scoliosis and clinodactyly. Clinical examination revealed typical facial dysmorphic features of ATS: broad forehead, triangular shape of the face, hypertelorism, microstomia, low-set ears, and mandibular retrognathism. Intraoral examination revealed: high-arched palate, crowding in the dental arches, hypomineralisation of enamel and high incidence of dental caries. Dental age assessment by Demirijan pointed to delayed development of permanent dentition. Cephalometric analysis revealed skeletal class II with high angle vertical jaws relation. Diagnosis of ATS requires high index of suspicion because of a great variability in the clinical manifestation of the syndrome. The subtle nature of the dysmorphic features often delays the diagnosis of this syndrome, and its potentially lethal cardiac arrhythmia remaining undetected.
\end{abstract}

\section{Key words}

Andersen-Tawil syndrome, dysmorphic features, craniofacial complex

\section{INTRODUCTION}

Andersen-Tawil syndrome (ATS) is a rare genetic disorder characterized by the classic triad of symptoms: ventricular arrhythmia associated with QT prolongation in electrograms (ECGs), periodic paralysis and dysmorphic skeletal and facial features. It is inherited in an autosomal dominant pattern, but ATS occurs sporadically resulting from a heterozygous mutation of KCNJ2 gene. The gene encodes the inward rectifier potassium channel Kir2.1 protein, highly expressed in the myocardium, skeletal muscle and brain. Kir2.1 also plays a major role in developmental signaling, accounting for the characteristic facial dysmorphic features $[1,2,3]$.

Probably the first mention of the syndrome was made in 1963 by Klein et al., who reported a particular form of periodic paralysis combined with premature ventricular contractions [1]. The first complete description of the syndrome with the characteristic triad of symptoms was made in 1971 by Ellen Damgaard Andersen et al. [2]. The syndrome was not correctly characterized until a number of patients were reported by Tawil et al. in 1994 [3]. In 2003, it was suggested naming the syndrome "Andersen-Tawil syndrome" in recognition for Tawil's contribution to the syndrome.

The exact prevalence of ATS is unknown, but estimates of 1/1,000,000 have been made. By 2004, at least 104 ATS patients had been reported in the literature worldwide. To date, this number has probably doubled [4].

Address for correspondence: Katarzyna Olszewska, Chair and Department of Jaw Orthopaedics, Medical University in Lublin, Poland, Staszica 16, 20-081 Lublin, Poland

E-mail: catieol@interia.pl

Received: 22 July 2014; accepted: 28 November 2014
For the diagnosis of ATS it is important to note that not all ATS patients present with the same symptoms, even within a single family. There are mutation carriers who are completely non-penetrant for the mutation and patients who show, in all possible combinations, one, two or three of the classic triad of ATS symptoms. The diagnosis of ATS can be established in the presence of two of the following three symptoms: 1) periodic paralysis, 2) KCNJ2 mutation with electrocardiographic abnormalities (enlarged U-waves, ventricular ectopy, nonsustained ventricular tachycardia or a prolonged QTc interval), and 3) characteristic facial and skeletal dysmorphic features (at least two). A patient with only one of the features mentioned above can be diagnosed with ATS on condition that there is one family member with an established diagnosis $[3,5,6]$.

The dysmorphic features are highly variable and include short stature, scoliosis, clinodactyly, syndactyly, brachydactyly, tapering fingers, hypertelorism, mandibular hypoplasia, lowset ears, malar hypoplasia, broad nasal root, micrognathia, ptosis, cleft palate, high-arched palate, broad forehead, thin upper lip, triangular shape of the face, and dental abnormalities (delayed tooth eruption, persistant primary dentition, oligodontia, dental crowding, enamel hypoplasia and discolorations) [7]. Novel findings include small hands and feet $(<10$ th centile for age) and joint laxity. The mechanism behind the skeletal and cranial dysmorphologies in ATS has not been elucidated. One theory mentions dysfunction of osteoclasts as a result of alterations in ion homeostasis. However, a loss-of-function mutation in KCNJ2 might impair the adequate functioning of osteoclasts by disrupting their ion balance. A problem with this theory is that the bone hypoplasia seen in ATS patients suggests an enhanced rather than impaired functioning of the osteoclasts [8]. 
Cardiac manifestations vary from an asymptomatic long QT syndrome to life-threatening ventricular tachyarrhythmia requiring an implantable defibrillator, but most patients exhibit either a long QTc interval or a prolonged QU interval with characteristic U-wave morphology [7]. Episodes of muscle weakness may fluctuate in severity and usually begin before the age of 10 years or in adolescence. Mild permanent weakness may be seen in some patients [9]. Recently, Yoon et al. colleagues described a distinct neurocognitive phenotype associated with ATS, characterized by deficits in abstract reasoning and executive dysfunction, suggesting that the clinical phenotypes of ATS may involve the central nervous system (CNS). These observations support previous studies that Kir2.1 has an important role in neural development [10].

Genetic testing may confirm the clinical diagnosis of ATS by revealing the presence of a pathogenic mutation in KCNJ2. In particular, mutational analysis could assist in the diagnosis of patients exhibiting only one of the ATS symptoms. It should, however, be kept in mind that a mutation in KCNJ2 cannot be identified in a minority of ATS patients. Type 1 of ATS, in which a mutation in KCNJ2 gene can be identified, accounts for about $60-70 \%$ of all ATS patients. The remaining $30-40 \%$ of ATS cases is designated as type 2 (ATS2), for which the genetic cause is still unknown $[11,12,13]$.

The aim of the study was to present characteristic features of the rare Andersen-Tawil syndrome (ATS) within the face and oral cavity of a 9-year-old boy.

\section{CASE REPORT}

A 9-year-old boy was admitted to the Chair and Department of Paediatric Dentistry, referred by a cardiologist, with the aim of performing complex dental treatment and prophylaxis. According to information from the genetics department, the patient was diagnosed with Andersen-Tawil syndrome (OMIM\#170390) at the age of 8 due to a positive family history, typical dysmorphic features, and the presence of mutation in the KCNJ2 gene confirmed by genetic testing. The family history of the patient revealed the prevalence of a prolongation of the QT interval, ventricular arrhythmia, short stature and typical dysmorphic features in his mother, maternal grandfather and his mothers' sisters. The boy was diagnosed with long QT syndrome, short stature, scoliosis, clinodactyly of the fifth finger and neurocognitive defects, and he remained under the specialist control of a neurologist concerning possible occurrence of periodic paralysis episodes and muscle weakness.

Clinical examination, extraoral and intraoral photographs (Fig. 1, 2, 3), orthopantomograph (Fig. 4) and a lateral cephalometric radiograph were performed (Fig. 5). The physical examination revealed typical facial dysmorphic features of ATS: broad forehead, triangular shape of the face, hypertelorism, microstomia, low-set ears, mandibular retrognathia and convex facial profile. Intraoral examination revealed: high-arched palate, crowding in the dental arches, hypomineralisation of the enamel of permanent teeth and high incidence of dental caries. Dental age assessment by Demirijan indicated delayed development of the permanent dentition. Cephalometric analysis by Segner and Hasund revealed skeletal class II with mandibular retrognathism and high angle vertical jaws relation.

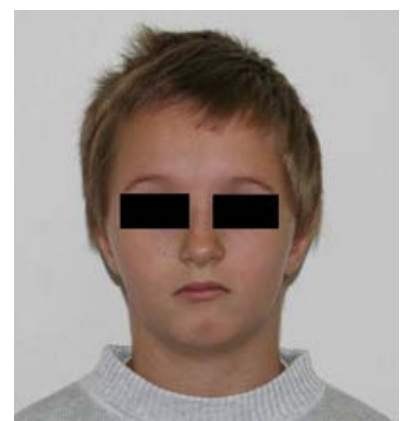

Figure 1. Extraoral photograph presenting characteristic facial features

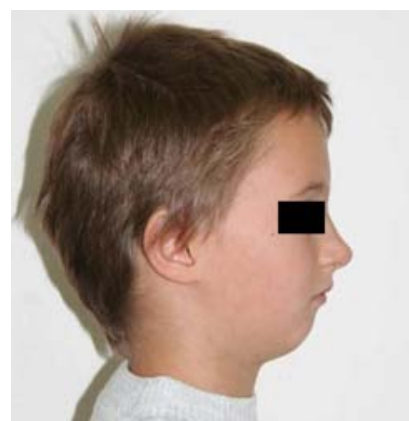

Figure 2. Extraoral photograph presenting convex facial profile pointing to the mandibular retrognathism

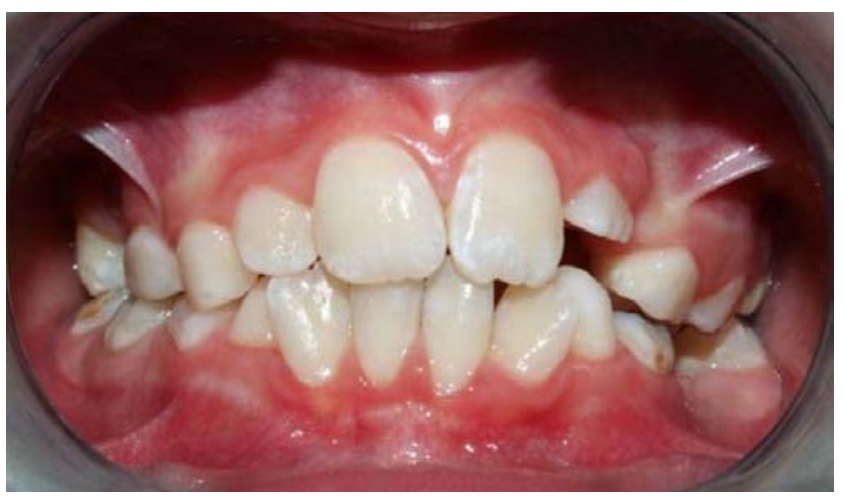

Figure 3. Intraoral photograph presenting dental arches in centric occlusion

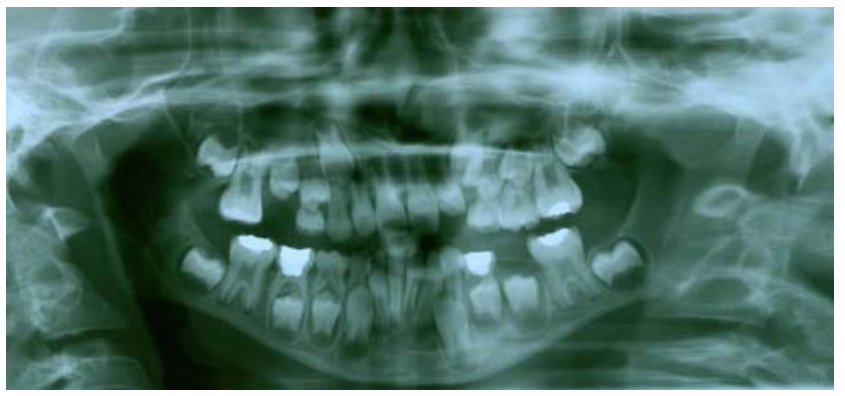

Figure 4. Orthopantomograph - assessment of dental age by Demirijan indicates delayed development of permanent dentition

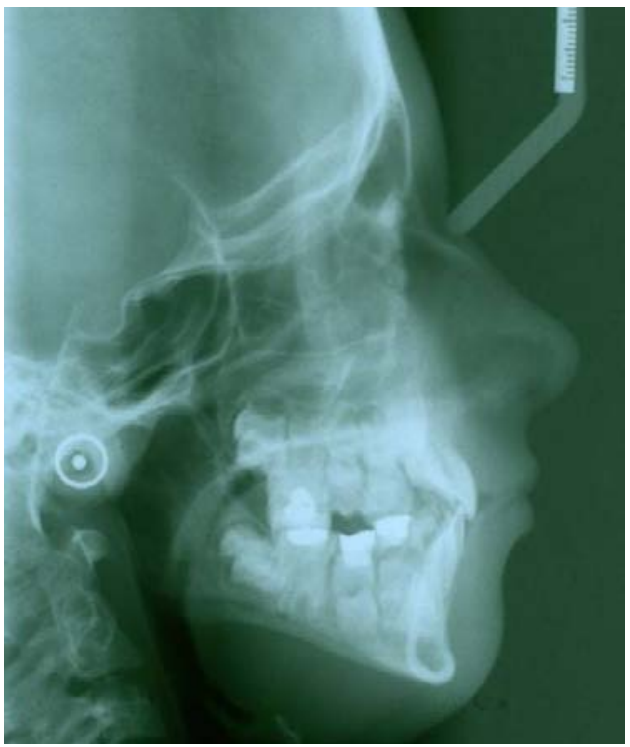

Figure 5. Lateral cephalometric radiograph - cephalometric analysis revealed skeletal class II with retrognathic mandible and high angle vertical jaws relation 


\section{DISCUSSION}

A set of symptoms unique to ATS with regard to the other types of Long QT Syndromes (LQTS) are dysmorphologies that can be observed in the majority of ATS patients.

In 1971, Andersen et al. [2] described a boy with the following clinical symptoms: low-set ears, broad nasal root, mandibular hypoplasia, hypertelorism, soft and hard palate defects, scaphocephaly, clinodactyly, short stature, ventricular extra-systoles, and attacks of muscular weakness.

Sansone et al. [5] described 11 patients from 5 kindreds with the classic triad of symptoms: potassium-sensitive periodic paralysis, ventricular arrhythmia and unusual facial features. Tristani-Firouzi et al. [6] presented a comprehensive clinical and in vitro study performed on a total of 17 kindreds with 10 different mutations. In the study, at least 2 dysmorphic features were diagnosed in 28 of 36 KCNJ2 mutation carriers: 14 of the 36 had low-set ears, 13 were diagnosed with hypertelorism, in 16 of the 36 small mandibles were reported, 23 had clinodactyly, and 4 were diagnosed with syndactyly. Cleft palate was observed in 3 of 36 ATS individuals and scoliosis in 4 of them. Dysmorphic features were in the majority of cases mild and non-disfiguring and could easily have been overlooked in routine clinical examination. Andelfinger et al. [14] assessed a large kindred in which ventricular arrhythmia (in female members) and periodic paralysis (in male members) segregated as autosomal dominant traits. In some mutation carriers, dysmorphic features were reported, including hypertelorism, syndactyly, clinodactyly, mandibular hypoplasia, cleft palate and scoliosis, which combined with cardiodysrhythmic periodic paralysis, have been named the 'Andersen syndrome'. Yoon et al. [7] prospectively examined 10 individuals with genetically confirmed mutations in the KCNJ2 gene, and observed a characteristic pattern of typical craniofacial features, as well as dental and skeletal anomalies. The characteristic craniofacial features included broad forehead, short palpebral fissures, malar, maxillary and mandibular hypoplasia, thin upper lip, high-arched or cleft palate, triangular shape of the face, relatively long nose with fullness along the bridge and a bulbous tip, and mild facial asymmetry. Dental anomalies were diagnosed in all patients and included delayed eruption of permanent dentition, oligodontia, and dental root anomalies. Skeletal anomalies included small hands and feet, brachydactyly, 2-3 toe syndactyly and toe clinodactyly. Tengan et al. [15] described a 33-year-old Brazilian male with episodic weakness and facial and skeletal dysmorphic features, such as clinodactyly of the fourth and fifth fingers, short stature, thoracic scoliosis, high arched palate, micrognathia and retrognathia. Detection of a mutation in the KCNJ2 gene confirmed the diagnosis of ATS. The mutation was also found in a 6-year-old daughter, who was diagnosed with such dysmorphic anomalies as micrognathia, clinodactyly of the fourth and fifth fingers, and short stature. The other three relatives of the patient presented only a fifth finger clinodactyly.

Haruna et al. [16] observed at least two dysmorphic features in 17 of their 23 patients (74\%), with mandibular micrognathia most frequently observed $(11 ; 48 \%)$. Short stature was found in 8 (35\%); clinodactyly in $6(26 \%)$; hypertelorism in $6(26 \%)$; low-set ears in 5 (22\%); broad forehead in 4 (17\%); and scoliosis in 1 (4\%). Kotulska and Kucharz [17] described a patient with suspicion of Andersen-Tawil syndrome on the basis of such clinical symptoms as periodic muscular weakness, cardiac abnormalities and micrognathia. The presence of elevated creatine kinase activity in the father of the patient was an additional significant suggestion for the diagnosis. Kamate et al. [18] reported the case of a 14-year-old boy with periodic paralysis, prolonged QTc interval, short stature and facial dysmorphic features like micrognathia, retrognathia, clinodactyly of the fifth fingers, hypertelorism and high arched palate. Chan et al. [19] reported the case of a 35-year-old woman who presented with episodic paralysis, typical ATS facial and skeletal features, including short stature, broad forehead, ocular hypertelorism, small head size, low-set ears, mandibular hypoplasia and clinodactyly of the fifth fingers and toes, as well as transient ventricular bigeminy and prolonged QT interval, fulfilling the criteria for long-QT syndrome. Her case was later diagnosed as major depression at the age of 35 years, in addition to the clinical triad of ATS. Barajas-Martinez [20] reported a novel de novo KCNJ2 mutation resulting in classical phenotypic features of ATS as well as polymorphic ventricular tachycardia mimicry in a 10 -year-old female patient. The patient presented facial and skeletal dysmorphic features such as micrognathia and retrognathia, broad-based nose, hypertelorism, clinodactyly of the third digit, and syndactyly of the second, third and fourth toes; moreover, syncope, frequent ventricular extrasystoles, non-sustained polymorphic ventricular tachycardia (PVT), BiVT, and prolonged QTc, secondary to a novel de novo mutation in KCNJ2. Thakkar et al. [21] described a 19-year-old patient with a suspected AndersenTawil syndrome with the clinical triad of periodic paralysis, ventricular arrhythmia and dysmorphic features: short stature, low set ears, micrognathia and retrognathia. His younger brother also had similar dysmorphic features and ventricular extra-systole. Fernlund et al. [22] described a 5 -generation family in which 10 of 21 individuals appeared to be KCNJ2 mutation carriers. All KCNJ2 affected family members showed dysmorphic features characteristic for ATS. All of them were of short stature, had small mandibles, dental engagement with enamel hypoplasia, and were prone to caries. Missing or ectopic teeth were noted in 7 of the 10 individuals. Cleft palate was found in three. Toe-syndactylia was seen in 3 of the affected individuals. Digit clinodactylia was seen in all mutation carriers. Muscular weakness was noted in only 2 members and only 1 family member had periodic paralysis. Life-threatening ventricular arrhythmias were observed during childhood in 5 of the 10 mutation carriers.

Table 1 presents an overview of the most prevalent facial dysmorphic features reported in the literature worldwide. It is the presence of these traits, however subtle, which is often the clue to the diagnosis. In accordance with classical descriptions of ATS phenotypes, dysmorphic features could be recognized from early childhood, but the cardiac symptoms do not occur until 7 years of age, or later. Data on the dental phenotype of ATS patients are limited. Yet, dental malformations can be expected, as the locus of KCNJ2 appears to be linked with tooth development. The most frequently observed dental problems include delayed tooth eruption, persistent primary dentition, oligodontia, dental crowding, enamel hypoplasia and discolourations [7]. 
Table 1. Facial dysmorphism in Andersen-Tawil syndrome (ATS)

\begin{tabular}{|c|c|c|}
\hline Author & Year & Facial dysmorphologies \\
\hline Andersen et al. ${ }^{2}$ & 1971 & $\begin{array}{l}\text { low-set ears, hypertelorism, broad nasal root, } \\
\text { mandibular hypoplasia, soft and hard palate } \\
\text { defects, scaphocephaly }\end{array}$ \\
\hline Sansone et al. ${ }^{5}$ & 1997 & $\begin{array}{l}\text { broad nose, broad forehead, high-arched or cleft } \\
\text { palate, hypertelorism, low-set ears, mandibular } \\
\text { hypoplasia }\end{array}$ \\
\hline Tristani-Firouzi et al. ${ }^{6}$ & 2002 & $\begin{array}{l}\text { high-arched or cleft palate, hypertelorism, low- } \\
\text { set ears, mandibular hypoplasia, }\end{array}$ \\
\hline Andelfinger et al. ${ }^{14}$ & 2002 & hypertelorism, small mandible, cleft palate \\
\hline Yoon et al. ${ }^{7}$ & 2006 & $\begin{array}{l}\text { broad forehead, high-arched or cleft palate, } \\
\text { hypertelorism, low-set ears, mandibular } \\
\text { hypoplasia }\end{array}$ \\
\hline Tengan et al. ${ }^{15}$ & 2006 & micrognathia, retrognathia, arched palate \\
\hline Haruna et al. ${ }^{16}$ & 2007 & $\begin{array}{l}\text { broad forehead, hypertelorism, low-set ears, } \\
\text { mandibular hypoplasia }\end{array}$ \\
\hline Kotulska and Kucharz ${ }^{17}$ & 2008 & micrognathia \\
\hline Kamate et al. ${ }^{18}$ & 2009 & $\begin{array}{l}\text { micrognathia, retrognathia, hypertelorism, high } \\
\text { arched palate }\end{array}$ \\
\hline Chan et al. ${ }^{19}$ & 2010 & $\begin{array}{l}\text { broad forehead, hypertelorism, small head size, } \\
\text { low-set ears, mandibular hypoplasia }\end{array}$ \\
\hline Barajas-Martinez ${ }^{20}$ & 2011 & $\begin{array}{l}\text { micrognathia and retrognathia, hypertelorism, } \\
\text { broad-based nose }\end{array}$ \\
\hline Thakkar et al. ${ }^{21}$ & 2012 & low set ears, micrognathia and retrognathia. \\
\hline Fernlund et al. ${ }^{22}$ & 2013 & $\begin{array}{l}\text { small mandible, dental engagement with enamel } \\
\text { hypoplasia, missing or ectopic teeth, cleft palate }\end{array}$ \\
\hline
\end{tabular}

\section{CONCLUSIONS}

The diagnosis of ATS requires a high index of suspicion because of the great variability in the clinical manifestation of the syndrome. The subtle nature of the dysmorphic features often delays the diagnosis of this syndrome, and its potentially lethal cardiac arrhythmia remaining undetected. Dental professionals may be the first physicians to identify unrecognized facial dysmorphism suggesting a genetic disorder, and refer the patient for further examination and investigation. Thus, it is very important to have the knowledge and awareness of the clinical manifestation of the rare Andersen-Tawil syndrome within the craniofacial complex.

\section{REFERENCES}

1. Klein R, Ganelin R, Marks JF, Usher P, Richards C. Periodic paralysis with cardiac arrhythmia. J Pediatr. 1963; 62: 371-385.

2. Andersen ED, Krasilnikoff PA, Overvad H. Intermittent muscular weakness, extrasystoles and multiple developmental anomalies. A new syndrome? Acta Paediatr Scand. 1971; 60: 559-564.

3. Tawil R, Ptacek LJ, Pavlakis SG. Andersen's syndrome: potassiumsensitive periodic paralysis, ventricular ectopy, and dysmorphic features. Ann Neurol. 1994; 35: 326-330.

4. Nguyen HL, Pieper GH, Wilders R. Andersen-Tawil syndrome: clinical and molecular aspects. Int J Cardiol. 2013; 170: 1-16.

5. Sansone V, Griggs RC, Meola G. Andersen's syndrome: a distinct periodic paralysis. Ann Neurol. 1997; 42: 305-312.

6. Tristani-Firouzi M, Jensen JL, Donaldson MR. Functional and clinical characterization of KCNJ2 mutations associated with LQT7 (Andersen syndrome). Clin Invest. 2002; 110: 381-388.

7. Yoon G, Oberoi S, Tristani-Firouzi M. Andersen-Tawil syndrome: prospective cohort analysis and expansion of the phenotype. Am J Med Genet A. 2006; 140: 312-321.

8. Donaldson MR, Yoon G, Fu YH, Ptacek LJ. Andersen-Tawil syndrome: a model of clinical variability, pleiotropy, and genetic heterogeneity. Ann Med. 2004; 36 (Suppl. 1): 92-97.

9. Sansone V, Tawil R. Management and treatment of Andersen-Tawil syndrome (ATS). Neurotherapeutics. 2007; 4: 233-237.

10. Yoon G, Quitania L, Kramer JH, Fu YH, Miller BL, Ptácek LJ. Andersen-Tawil syndrome: definition of a neurocognitive phenotype. Neurology. 2006; 66: 1703-1710.

11. Plaster NM, Tawil R, Tristani-Firouzi M. Mutations in Kir2.1 cause the developmental and episodic electrical phenotypes of Andersen's syndrome. Cell. 2001; 105: 511-519.

12. Limberg MM, Zumhagen S, Netter MF. Non dominant-negative KCNJ2 gene mutations leading to Andersen-Tawil syndrome with an isolated cardiac phenotype. Basic Res Cardiol. 2013; 108: 353.

13. Hasegawa K, Ohno S, Kimura H. Mosaic KCNJ2 mutation in Andersen-Tawil syndrome: targeted deep sequencing is useful for the detection of mosaicism. Clin Genet. 2014

14. Andelfinger G, Tapper AR, Welch RC. KCNJ2 mutation results in Andersen syndrome with sex-specific cardiac and skeletal muscle phenotypes. Am J Hum Genet. 2002; 71: 663-668.

15. Tengan CH, Antunes AC, Bauab JR, Prado GF, Manzano GM, Gabbai AA. Andersen syndrome: an association of periodic paralysis, cardiac arrhythmia and dysmorphic abnormalities. Arq Neuropsiquiatr. 2006; 64: 582-584.

16. Haruna Y, Kobori A, Makiyama T. Genotype-phenotype correlations of KCNJ2 mutations in Japanese patients with Andersen-Tawil syndrome. Hum Mutat. 2007; 28: 208.

17. Kotulska A, Kucharz EJ. Andersen-Tawil syndrome - a review of the literature and a case report. Reumatologia. 2008; 46(3): 171-174.

18. Kamate M, Chetal V. Andersen-Tawil syndrome - periodic paralysis with dysmorphism. Ind Pediatr. 2011; 48: 64-65.

19. Chan HF, Chen ML, Su JJ, Ko LC, Lin CH, Wu RM. A novel neuropsychiatric phenotype of KCNJ2 mutation in one Taiwanese family with Andersen-Tawil syndrome. J Hum Genet. 2010; 55: 186-188.

20. Barajas-Martinez H, Hu D, Ontiveros G. Biophysical and molecular characterization of a novel de novo KCNJ2 mutation associated with Andersen-Tawil syndrome and catecholaminergic polymorphic ventricular tachycardia mimicry. Circ Cardiovasc Genet. 2011; 4: 51-57.

21. Thakkar M, Biswas TK, Desle HB. Hypokalemic periodic paralysis, facial dysmorphism and ventricular arrhythmia (clinical triad of Andersen- Tawil syndrome). J Assoc Physician I. 2012; 60: 56-58.

22. Fernlund E, Lundin C, Hertervig E, Kongstad O, Alders M, Platonov P. Novel mutation in the KCNJ2 gene is associated with a malignant arrhythmic phenotype of Andersen-Tawil syndrome. Ann Noninvasive Electrocardiol. 2013; 18: 471-478. 